\title{
Uso de álcool e condições de saúde de motoristas de caminhão
}

\author{
Alcohol use and health conditions among truck drivers \\ Uso de alcohol y condiciones de salud de conductores de camión
}

\author{
Josélia Benedita Carneiro Domingos ${ }^{\text {I }}$ Sandra Cristina Pillon ${ }^{\text {II }}$; Manoel Antônio dos Santos ${ }^{\text {III; }}$ \\ Ronildo Alves dos Santos ${ }^{I V}$; Natália Prioli Jora ${ }^{V}$; Paulo Sérgio Ferreira ${ }^{\text {VI }}$
}

\begin{abstract}
RESUMO: O estudo teve por objetivo identificar o uso do álcool e as condições de saúde de motoristas de caminhão participantes de uma campanha de saúde. O desenho metodológico é do tipo descritivo, na abordagem quantitativa. A coleta de dados foi realizada, entre agosto e outubro de 2006, em uma rodovia do interior paulista, por meio de um questionário contendo informações sociodemográficas, Teste de Identificação do Uso de Álcool (AUDIT) e informações sobre condições de saúde. De 1087 participantes, 827 eram motoristas de caminhão, predominantemente do sexo masculino, de cor branca, casados, com baixo nível de escolaridade, católicos. Os resultados apontaram um conjunto de riscos associados em motoristas que pontuaram acima de 8 pontos no AUDIT, além de apresentarem níveis pressóricos, glicêmicos e de colesterol acima dos valores normais, bem como sobrepeso e obesidade. A identificação precoce dos problemas é uma alternativa válida para a aplicação de ações preventivas para essa população.
\end{abstract}

Palavras-Chave: Transtornos relacionados ao uso de álcool; promoção da saúde; enfermagem; motoristas.

\begin{abstract}
This study aimed to identify the alcohol use and the health condition of truck drivers participating in a health campaign. The methodological design is an descriptive of the quantitative approach. Data collection was conducted between August and October 2006 on a highway in São Paulo State, Brazil, by means of a questionnaire containing demographic information, Alcohol Use Disorders Identification Test (AUDIT) and health conditions issues. Among 1087 participants, 827 were truck drivers, predominantly male, adult, white, and married, with low level of education and Catholic religion. The results pointed out a list of risks among drivers who scored more than eight points in the AUDIT, who had high blood pressure, glucose and cholesterol levels above normal, and overweight and obesity. Early identification of both problems is a valid alternative to the implementation of preventive actions to this population.
\end{abstract}

Keywords: Alcohol-related disorders; health promotion; nursing; drivers.

RESUMEN: El estudio tuvo como objetivo identificar el consumo de alcohol y las condiciones de salud de los conductores de camiones que participan en una campaña de salud. El diseño del estudio es de tipo descriptivo, en el enfoque cuantitativo. La recolección de datos se llevó a cabo, entre agosto y octubre de 2006, en una carretera en São Paulo, Brasil, por medio de un cuestionario que contiene información sociodemográfica, Test de Identificación de Trastornos debido al Consumo de Alcohol (AUDIT) e informaciones sobre las condiciones de salud. De 1087 participantes, 827 eran conductores de camiones, predominantemente masculinos, blancos, casados, con bajo nivel de educación y religión católica. Los resultados mostraron puntuaciones de riesgos asociados a los conductores que obtuvieron una puntuación por encima de 8 puntos en la prueba de AUDIT, además de presentar los niveles de presión arterial, glucosa y colesterol por encima del normal, y sobrepeso y obesidad. La identificación temprana de los problemas es una alternativa válida para la aplicación de medidas preventivas para esa población.

Palabras Clave: Trastornos relacionados con alcohol; promoción de la salud; enfermería; conductores.

\section{INTRODUÇÃo}

A literatura traz bem documentado o papel do consumo de álcool no desencadeamento de diversas condições crônicas de saúde, o que vem ocorrendo de forma crescente devido à transição nutricional e epidemiológica. Apesar do consumo do álcool ser considerado um fator que potencializa a probabilidade do desenvolvimento de doenças crônicas não transmissíveis (DCNTs), essa área tem recebido pouca

'Enfermeira. Doutora em Ciências pelo Programa de Pós-Graduação em Enfermagem Psiquiátrica. Escola de Enfermagem de Ribeirão Preto. Universidade de São Paulo. Ribeirão Preto, São Paulo, Brasil. E-mail: joselia.domingos@usp.br

IIProfessora Titular. Departamento de Enfermagem Psiquiátrica e Ciências Humanas. Escola de Enfermagem de Ribeirão Preto. Universidade de São Paulo. Ribeirão Preto, São Paulo, Brasil. E-mail: pillon@eerp.usp.br

IIIProfessor Associado 3. Departamento de Psicologia. Faculdade de Filosofia, Ciências e Letras de Ribeirão Preto. Universidade de São Paulo. Ribeirão Preto, São Paulo, Brasil. E-mail: masantos@ffclrp.usp.br

${ }^{\text {IV }}$ Professor Doutor. Departamento de Enfermagem Psiquiátrica e Ciências Humanas da Universidade de São Paulo. Escola de Enfermagem de Ribeirão Preto. Universidade de São Paulo. Ribeirão Preto, São Paulo, Brasil. E-mail: ronildo@eerp.usp.br

vEnfermeira. Doutora em Ciências pelo Programa de Pós-Graduação em Enfermagem Psiquiátrica. Escola de Enfermagem de Ribeirão Preto. Universidade de São Paulo. Ribeirão Preto, São Paulo, Brasil. E-mail: natalia_jora@hotmail.com

VIEnfermeiro. Mestre. Departamento de Enfermagem Psiquiátrica e Ciências Humanas. Escola de Enfermagem de Ribeirão Preto. Universidade de São Paulo. Ribeirão Preto, São Paulo, Brasil. E-mail: pausefer@eerp.usp.br 
atenção, quando avaliada sob a ótica da cronicidade e, principalmente, da necessidade de identificação precoce dos fatores de risco para essas doenças e possíveis intervenções preventivas ${ }^{1-3}$.

Existe uma disjunção entre danos causados pelo uso do álcool e as iniciativas de controle ou manejo efetivo dos potenciais prejuízos. Profissionais e a população, de modo geral, ainda estão pouco conscientes acerca das consequências mais graves do uso abusivo do álcool, como se observa na situação do beber e dirigir. As consequências a médio e longo prazo, como as DCNTs relacionadas ao consumo de álcool, têm sido pouco investigadas. Tanto na pesquisa clínica, como na assistência e na formulação de políticas públicas de saúde destinadas às DCNTs, o enfoque privilegiado tem sido a identificação de fatores de risco relacionados ao uso de substâncias psicoativas (sobretudo álcool e tabaco) e estilo de vida (atividades físicas e padrões alimentares saudáveis) ${ }^{1}$.

Em 2008 foi promulgada a Lei 11.705/2008, que estabeleceu alcoolemia zero para o condutor de veículo no Brasil. Após a adoção desta lei, constatou-se uma redução imediata nas taxas de acidentes de trânsito 4 . O exame da alcoolemia tem sido foco de intensa discussão na mídia brasileira como principal estratégia para se promover uma efetiva redução da morbimortalidade no trânsito em decorrência do binômio álcool e direção. No entanto, há escassez de estudos que apontem as relações entre consumo e as DCNTs. A investigação desse tipo de consequência é relevante, principalmente, quando as DCNTs estão instaladas e as condições clínicas encontram-se agravadas, o motorista provavelmente já está incapacitado para dirigir. Nesses casos, as medidas preventivas de controle, com foco na relação direção e bebida alcoólica, não serão mais eficazes.

Considerando a necessidade de buscar evidências que permitam elucidar a relação entre consumo de álcool e saúde, o presente estudo teve por objetivo identificar o uso do álcool e as condições de saúde em motoristas de caminhão.

\section{REVISÃO DE LITERATURA}

A relação entre uso do álcool e DCNTs constitui um dos maiores problemas de saúde pública global. Consiste na principal causa de mortalidade e incapacidade, representando grave ameaça à saúde e ao desenvolvimento humano. Dados da Organização Mundial da Saúde $(\mathrm{OMS})^{2}$ apontam que, em nível mundial, o uso do álcool responde por 3,2\% de todas as mortes e $4 \%$ de todas as doenças ${ }^{3,5}$, o que implica custos sociais e econômicos elevados. Segundo a OMS, existe uma relação causal entre consumo do álcool e mais de 60 tipos de doenças ${ }^{1-3,5}$, incluindo condições cardiovasculares, gastrointestinais, neuropsiquiátricas e uma variedade de cânceres. O papel que o álcool ex- erce no desenvolvimento de DCNTs é particularmente importante à luz dos hábitos de consumo observados em diversos grupos da população, o que indica a relevância de se considerar esse nexo causal quando são planejadas políticas e medidas de enfrentamento, no âmbito da prevenção e tratamento 5 .

Nas últimas décadas, o consumo de álcool vem aumentando em diversos países, sendo que a maior parte desse incremento vem acontecendo nos países em desenvolvimento. Não apenas no Brasil, o álcool $(6,2 \%)$, o tabaco (2,0\%), a hipertensão arterial $(2,5 \%)$, o colesterol alterado (1,9\%), o Índice de Massa Corporal (IMC) $(2,7 \%)$ e o sobrepeso com alto índice de mortalidade $(14,9 \%)$ constituem os fatores que contribuem para o aumento dos agravos de saúde incapacitantes ${ }^{6-9}$.

Em termos conceituais o alcoolismo caracteriza-se como uma doença crônico-degenerativa, que compreende um conjunto de sinais e sintomas com altos índices de recaída. A severidade se desenvolve ao longo de um continuum e pode aparecer repentinamente ou se instalar de forma insidiosa ao longo do tempo?.

Além disso, as DCNT são aquelas que demandam um tempo para se desenvolver, são de longa duração e exigem o manejo de múltiplas estratégias de intervenção. Essas condições demandam um tratamento por tempo prolongado e geram sobrecarga no sistema de saúde. Nota-se também que vários dos prejuízos resultantes dessas condições são irreversíveis, podendo exigir um continuum de cuidados e reabilitação prolongada ${ }^{1-3}$.

As DCNTs também têm sido descritas como doenças do estilo de vida característico da sociedade contemporânea, desencadeadas por fatores de risco múltiplos e complexos, relacionados a áreas como: comportamento alimentar, condições de trabalho, atividade física, padrão do sono, uso de medicamentos, manejo do estresse, uso de drogas (álcool e tabaco), comportamentos de alto risco (exposição a acidentes e situações de violência) e relações familiares e sociais disfuncionais ${ }^{8,9}$.

Nos últimos anos, no contexto brasileiro questões relacionadas ao uso de álcool e aspectos relacionados à saúde têm sido enfatizadas ao se considerar o grupo de motoristas, principalmente os de caminhão $0^{10-17}$. Esse grupo de profissionais se destaca pela dificuldade de manutenção de um estilo de vida saudável, o que os coloca em situação de alta vulnerabilidade psicossocial, em virtude da longa jornada de trabalho e do pouco tempo disponível para realizar atividade física. A combinação desses fatores favorece o desenvolvimento do sobrepeso, obesidade e outras DCNTs ${ }^{10,11,17}$.

Estudos nesse grupo da população foram desenvolvidos com o intuito de identificar os fatores de risco, associando o uso de álcool, tabaco e anfetaminas 
às condições de saúde, tais como pressão arterial, glicemia, colesterol, triglicérides, sobrepeso e obesidade $^{10,14-17}$. Nesse contexto, a literatura sobre o uso do álcool e os aspectos relacionados às condições de saúde de motoristas de caminhão vêm mostrando índices alarmantes e preocupantes. Uma boa notícia é que a maioria dos fatores de risco constitui comportamentos modificáveis, ou seja, passíveis de ações preventivas.

Mediante o exposto, torna-se relevante a identificação do uso de álcool e das condições de saúde em motoristas de caminhão, de modo a contribuir para a formulação de políticas púbicas e programas preventivos em relação ao uso de substâncias psicoativas e aos agravos decorrentes do consumo crônico. Além disso, o estudo subsidiar o enfermeiro em suas atividades de prevenção, ajudando-o a delinear seu papel e suas atividades assistenciais nessa área do conhecimento, de modo a inseri-lo como profissional atuante, que participa de forma autônoma, individualizada ou em parceria com outros profissionais de saúde, na prevenção do uso de álcool e na promoção de saúde junto a motoristas de caminhão.

\section{Metodologia}

O desenho metodológico do estudo é do tipo descritivo, com abordagem quantitativa. A presente investigação foi desenvolvida no âmbito de um projeto de extensão universitária, que envolve a realização de uma campanha preventiva de saúde destinada a condutores de veículos, que ocorre trimestralmente em uma rodovia do interior paulista.

Dos 1.186(100\%) participantes atendidos em seis dias de duas campanhas de saúde na estrada, realizadas entre agosto e outubro de 2006, foram entrevistados $1.014(85,5 \%)$ motoristas que aceitaram participar da pesquisa. Destes, foram selecionados $827(81,5 \%)$ motoristas de caminhão para compor a amostra do presente estudo.

Os indivíduos foram selecionados conforme critérios de elegibilidade preestabelecidos, a saber: idade $\geq 18$ anos; ser motorista de caminhão e ser participante da campanha de saúde na estrada. Como critérios de exclusão: idade $\leq 18$ anos, não ser motorista de caminhão.

Para a coleta de dados, utilizou-se um questionário com perguntas fechadas, contendo informações sociodemográficas e o Teste para Identificação de Problemas Relacionados ao Uso do Álcool (AUDIT) composto por 10 perguntas que avaliam os níveis de risco em relação ao beber ${ }^{17}$. Informações sobre os aspectos relacionados à saúde, como a presença de doenças crônicas (hipertensão arterial severa, Diabetes Mellitus), colesterol, uso de medicação, e IMC. Dessa forma, considerou-se com peso normal o participante com um IMC menor que 25; obesas, as pessoas que apresentaram IMC igual ou superior a 30 $\mathrm{Kg} / \mathrm{m} 2$; e sobrepeso, aqueles com IMC entre 25 a $<30$ $\mathrm{Kg} / \mathrm{m} 2$, de acordo com a OMS ${ }^{11-14}$. A coleta de dados foi realizada pelos autores e a avaliação do IMC por um técnico habilitado.

O AUDIT é um instrumento de rastreamento do uso problemático de álcool que foi desenvolvido pela $\mathrm{OMS}^{18}$. Esse teste é muito utilizado em diversos países por ser de fácil aplicação e baixo custo. $\mathrm{O}$ escore total varia de zero a 40 pontos, que identifica dois níveis de uso de álcool ou zonas de risco, ou seja, pontuação $\leq$ 7 abstêmios ou de baixo risco e $\geq 8$ uso de risco ou problemático.

Este estudo seguiu as normas e procedimentos éticos de acordo com a Resolução no 196/96. O projeto foi aprovado pelo Comitê de Ética em Pesquisa da Escola de Enfermagem de Ribeirão Preto, Universidade de São Paulo (Processo no 033/2007).

Para a análise estatística, elaborou-se um banco de dados no programa Statistical Package for Social Science (SPSS) version 17 for Windows e Stata 7.0; consistiu da descrição da amostra, análise por meio da regressão logística (Odds ratio OR bruto e Odds ratio ORA ajustado), para avaliar as associações de risco entre consumo de bebidas alcoólicas, informações sociodemográficas e condições de saúde. Evidências de associação podem ser observadas se o valor um não estiver contido no intervalo com 95\% de confiança (IC 95\%).

\section{Resultados}

A amostra foi composta por 827 (81,5\%) motoristas de caminhão, com idade variando entre 21 a 72 anos, média de 41,3 $\pm 9,8$ anos, predominamente do sexo masculino -821 (99,3\%), casados/amasiados - 707 $(85,5 \%)$, de cor branca $-551(66,7 \%)$, com baixo nível de escolaridade (Ensino Fundamental completo ou incompleto) $-554(67 \%)$, professavam a religião católica $-584(70,6 \%)$, dados não apresentados em tabela.

As variáveis como faixa etária de 30 a 45 anos, religião católica, não ter religião e prática religiosa foram identificadas como preditoras de fatores de risco para o uso de álcool (AUDIT $\geq 8$ ) em motoristas de caminhão, com valores estatisticamente significantes. No entanto, outras variáveis como cor da pele, estado civil e escolaridade não apresentaram valores significantes nesta amostra. Chama a atenção a variável sexo feminino. Embora haja uma pequena parcela de mulheres na amostra, foi identificado como um grupo de potencial fator de risco, pois a maioria apresentou pontuação $\geq 8$ no AUDIT. Ver Tabela 1 .

Indivíduos que professavam a religião católica, espíritas ou que não tinham religião, quando comparados aos evangélicos, apresentaram pontuação $\geq 8$ no AUDIT, com razões de chances na ordem de 3,09; 2,49 
TABELA 1: Regressão logística entre informações sociodemográficas e a pontuação no AUDIT dos motoristas de caminhão, Campanha Saúde na Estrada. Ribeirão Preto, SP, 2006. (N=827).

\begin{tabular}{|c|c|c|c|c|c|c|}
\hline \multirow[b]{2}{*}{ Variáveis } & \multicolumn{4}{|c|}{ AUDIT } & \multirow{2}{*}{$\begin{array}{l}\text { OR (IC 95\%) } \\
\text { Bruto (ORB) }\end{array}$} & \multirow{2}{*}{$\begin{array}{c}\text { OR (IC 95\%) } \\
\text { Ajustado (ORA) }\end{array}$} \\
\hline & $f$ & $\%$ & $f$ & $\%$ & & \\
\hline \multicolumn{7}{|l|}{ Idade } \\
\hline $30 \operatorname{anos}(*)$ & 66 & 54,6 & 55 & 45,4 & $1,78(0,68 ; 4,69)$ & $3,04(1,04 ; 8,84)$ \\
\hline$>30$ a $45(*)$ & 221 & 52,1 & 203 & 47,9 & $1,96(0,78 ; 4,92)$ & $3,46(1,28 ; 9,36)$ \\
\hline$>45$ a 60 & 161 & 61,9 & 99 & 38,1 & $1,31(0,51 ; 3,34)$ & $1,98(0,73 ; 5,36)$ \\
\hline 60 anos & 15 & 68,2 & 7 & 31,8 & - & - \\
\hline \multicolumn{7}{|l|}{ Sexo } \\
\hline Masculino & 461 & 56,2 & 360 & 43,8 & - & - \\
\hline Feminino & 2 & 33,3 & 4 & 66,7 & $2,56(0,46 ; 14,06)$ & $3,00(0,48 ; 18,51)$ \\
\hline \multicolumn{7}{|l|}{ Religião } \\
\hline Católica $(*)$ & 290 & 49,7 & 294 & 50,3 & $3,97(2,66 ; 5,92)$ & $3,09(1,98 ; 4,80)$ \\
\hline Evangélica & 141 & 79,7 & 36 & 20,3 & - & $=$ \\
\hline Espírita $(*)$ & 11 & 57,9 & 8 & 42,1 & $2,84(1,06 ; 7,60)$ & $2,49(0,87 ; 7,11)$ \\
\hline Não tem $\left.{ }^{*}\right)$ & 21 & 44,7 & 26 & 55,3 & $4,84(2,45 ; 9,58)$ & $3,20(1,50 ; 6,79)$ \\
\hline \multicolumn{7}{|l|}{ Prática Religiosa } \\
\hline $\operatorname{Sim}(*)$ & 241 & 48,2 & 259 & 51,8 & $2,27(1,69 ; 3,03)$ & $1,55(1,10 ; 2,18)$ \\
\hline Não & 222 & 67,9 & 105 & 32,1 & - & - \\
\hline \multicolumn{7}{|l|}{ Cor de pele } \\
\hline Branco & 313 & 56,8 & 238 & 43,2 & $1,19(0,78 ; 1,82)$ & $1,04(0,66 ; 1,66)$ \\
\hline Negro & 66 & 61,1 & 42 & 38,9 & - & $=$ \\
\hline Pardo & 81 & 50,0 & 81 & 50,0 & $1,57(0,95 ; 2,57)$ & $1,31(0,754 ; 2,281)$ \\
\hline Amarelo & 3 & 50,0 & 3 & 50,0 & $1,57(0,30 ; 8,15)$ & $1,99(0,35 ; 11,29)$ \\
\hline \multicolumn{7}{|l|}{ Estado Civil } \\
\hline $\begin{array}{l}\text { Solteiros/divorciado } \\
\text { s/separados }\end{array}$ & 62 & 51,7 & 58 & 48,3 & $1,22(0,83 ; 1,80)$ & $1,17(0,76 ; 1,80)$ \\
\hline Casado /amasiado & 401 & 56,7 & 306 & 43,3 & - & - \\
\hline \multicolumn{7}{|l|}{ Escolaridade } \\
\hline Ensino & 322 & 58,1 & 232 & 41,9 & - & - \\
\hline Fundamental & & & & & & \\
\hline Ensino Médio & 134 & 51,7 & 125 & 48,3 & $1,295(0,96 ; 1,74)$ & $1,245(0,88 ; 1,74)$ \\
\hline Ensino Superior & 7 & 50,0 & 7 & 50,0 & $1,388(0,48 ; 4,01)$ & $1,743(0,53 ; 5,68)$ \\
\hline
\end{tabular}

$(*)$ valores de $\mathrm{p}<.0,05$

e 3,20, respectivamente. A variável pertencer à religião evangélica apresenta-se como um fator de proteção, pois um número considerável de participantes adeptos dessa crença religiosa foi classificado com pontuação $\leq$ 8 no AUDIT, conforme mostra a Tabela 1.

Dos motoristas de caminhão, $610(73,8 \%)$ fizeram uso de bebidas alcoólicas independentemente do nível de risco. Destaca-se que 265 (32\%) consumiram em níveis de baixo risco e 264 (31,9\%) fizeram uso de risco. Os dados estão apresentados na Tabela 2.

Com exceção do uso de medicamentos e da presença de doenças crônicas, embora não tenha havido associações estatisticamente significativas, o maior

TABELA 2: Níveis de risco relacionados ao consumo de álcool (AUDIT) dos motoristas de caminhão, Campanha Saúde na Estrada. Ribeirão Preto, SP, 2006. $(\mathrm{N}=827)$.

\begin{tabular}{lcc}
\hline Níveis de risco (AUDIT) & f & \% \\
\hline Abstêmios & 217 & 26,2 \\
Uso de baixo risco & 265 & 32,0 \\
Uso de risco & 264 & 31,9 \\
Uso nocivo & 59 & 7,1 \\
Provável dependência & 22 & 2,7 \\
\hline
\end{tabular}

risco esteve presente em motoristas que pontuaram $\geq 8$ no AUDIT, além da presença de doenças como: hipertensão, diabetes e colesterol acima dos níveis normais. Entre os fatores de risco, identificou-se associação entre AUDIT $\geq 8$, sobrepeso e obesidade, conforme apresentado na Tabela 3.

\section{Discussão}

Este estudo avaliou o uso de álcool e as condições de saúde em motoristas de caminhão participantes de uma campanha preventiva de saúde na estrada.

A amostra caracterizou-se predominantemente por indivíduos do sexo masculino, adultos, casados e/ ou em união consensual, de cor branca, com baixo nível de escolaridade e que professavam a religião católica. Esses achados são consistentes com os da literatura que avalia essa categoria profissiona ${ }^{10-17}$. Os estudos mostraram que há associação negativa entre o consumo abusivo do álcool e a situação socioeconômica, educação, ocupação e renda.

As características sociodemográficas identificadas entre os participantes do presente estudo corrobo- 
TABELA 3: Regressão logística entre pontuação no AUDIT e condições de saúde dos motoristas de caminhão participantes da Campanha Saúde na Estrada. Ribeirão Preto, SP, 2006. (N=827)

\begin{tabular}{|c|c|c|c|c|c|c|}
\hline \multirow{3}{*}{ Variáveis } & \multicolumn{4}{|c|}{ AUDIT } & \multirow{3}{*}{$\begin{array}{l}\text { OR (IC 95\%) } \\
\text { Bruto (ORB) }\end{array}$} & \multirow{3}{*}{$\begin{array}{c}\text { OR (IC 95\%) } \\
\text { Ajustado (ORA) }\end{array}$} \\
\hline & \multicolumn{2}{|c|}{7} & \multicolumn{2}{|c|}{8} & & \\
\hline & $f$ & $\%$ & $f$ & $\%$ & & \\
\hline \multicolumn{7}{|l|}{ Doenças Crônicas } \\
\hline Sim & 87 & 58,0 & 63 & 42,0 & - & - \\
\hline Não & 376 & 55,5 & 301 & 44,5 & $\begin{array}{c}1,10 \\
(0,77 ; 1,58)\end{array}$ & $1,34(0,35 ; 5,06)$ \\
\hline \multicolumn{7}{|l|}{ Medicação } \\
\hline Sim & 84 & 57,9 & 61 & 42,1 & - & - \\
\hline Não & 379 & 55,6 & 303 & 44,4 & $\begin{array}{c}1,10 \\
(0,76 ; 1,58)\end{array}$ & $0,78(0,21 ; 2,96)$ \\
\hline \multicolumn{7}{|c|}{ Hipertensão Arterial } \\
\hline Sim & 51 & 48,1 & 55 & 51,9 & $\begin{array}{c}1,43 \\
(0,95 ; 2,16)\end{array}$ & $1,47(0,92 ; 2,34)$ \\
\hline Não & 412 & 57,1 & 309 & 42,9 & - & - \\
\hline \multicolumn{7}{|l|}{ Diabetes } \\
\hline Sim & 33 & 54,1 & 28 & 45,9 & $\begin{array}{c}1,08 \\
(0,64 ; 1,83)\end{array}$ & $1,07(0,59 ; 1,93)$ \\
\hline Não & 430 & 56,1 & 336 & 43,9 & - & - \\
\hline \multicolumn{7}{|l|}{ Colesterol } \\
\hline Sim & 35 & 50,7 & 34 & 49,3 & $\begin{array}{c}1,26 \\
(0,76 ; 2,06)\end{array}$ & $1,02(0,59 ; 1,76)$ \\
\hline Não & 428 & 56,5 & 330 & 43,5 & - & - \\
\hline \multicolumn{7}{|l|}{ IMC } \\
\hline Normal & 150 & 64,4 & 83 & 35,6 & - & - \\
\hline Sobrepeso $(*)$ & 201 & 54,5 & 168 & 45,5 & $\begin{array}{c}1,51 \\
(1,07 ; 2,11)\end{array}$ & $1,70(1,16 ; 2,48)$ \\
\hline Obesos $(*)$ & 112 & 49,8 & 113 & 50,2 & $\begin{array}{c}1,82 \\
(1,25 ; 2,65)\end{array}$ & $1,85(1,23 ; 2,80)$ \\
\hline
\end{tabular}

$\left.{ }^{*}\right)$ valores de $\mathrm{p}<.0,05$

ram as propostas da campanha de saúde na estrada, que tem por objetivo atingir esse público e oferecer um trabalho preventivo, de promoção social e de saúde aos motoristas, principalmente os de caminhão ${ }^{10,12}$. Trata-se de um grupo considerado prioritário para o desenvolvimento de ações e estratégias preventivas e de promoção de saúde, pois pertence a uma parcela economicamente ativa do país, apresenta maior vulnerabilidade para desenvolver comportamentos de risco, DCNTs e doenças sexualmente transmissíveis (DSTs), uso de álcool, tabaco e outras drogas, com potenciais consequências deletérias para a saúde ${ }^{1,2}$.

De longa data a literatura aponta que existe uma relação complexa entre o consumo do álcool e as condições de saúde em motoristas ${ }^{12-17}$, que muitas vezes se apresentam precárias. Isso corrobora os dados da literatura, que apresentam a relação causal do uso crônico de bebidas alcoólicas com mais de 60 tipos de doenças5, como obesidade, hipertensão arterial e pobre controle do Diabetes Mellitus ${ }^{12-15}$.

Em relação ao consumo de bebidas alcoólicas, $73,8 \%$ dos motoristas fizeram uso, independentemente do nível de risco pontuado pelo AUDIT. Esses índices são considerados bastante elevados quando comparados a outros estudos brasileiros ${ }^{12-17}$. Quanto à classificação de níveis de risco em relação ao beber, 265(32\%) motoristas consumiram álcool em níveis de baixo risco e 264(31,9\%) apresentaram uso de risco, segundo a Tabela 2. Isso significa que os motoristas de caminhão estão consumindo bebidas alcoólicas em quantidades preocupantes, não obstante as organizações internacionais de saúde preconizarem que o beber sensato caracteriza-se por um consumo que não prejudica a si mesmo, não coloca em risco a própria saúde e tão pouco a de outros ${ }^{18}$.

Desse modo, pode-se questionar o quanto têm sido efetivas as medidas de controle da associação entre beber e dirigir, bem como as campanhas de prevenção do uso de álcool para motoristas. Apesar de o país contar com uma lei altamente coercitiva (Lei Seca em relação ao beber e dirigir), é preciso envidar esforços no sentido de uma fiscalização mais rigorosa nas estradas.

Há que se ressaltar que o uso do álcool tem sido um dos maiores problemas de saúde pública global ${ }^{1-3}$, não só para as pessoas que já desenvolveram a dependência alcoólica, mas principalmente para aquelas que estão fazendo uso em níveis de risco e nocivo. A maioria dos prejuízos (individuais, familiares e na comuni- 
dade) relacionada ao uso do álcool não é consequência da própria dependência do álcool, pois a porcentagem de dependentes em relação aos que fazem uso em níveis de risco é relativamente menor na população e muitas vezes já estão fora do mercado de trabalho devido à incapacitação gerada pelo alcoolismo. Geralmente, os problemas existentes aparecem mais entre os que bebem em níveis de risco na população, associados aos diferentes padrões de mortalidade e morbidade ${ }^{18}$. Dessa maneira, o foco das campanhas e de outras estratégias preventivas deveria ser direcionado ao seguimento de consumidores em níveis de risco e nocivo.

A compreensão do impacto do uso do álcool sobre as condições de saúde em motoristas de caminhão implica múltiplos desafios, lembrando que problemas relacionados ao álcool não resultam apenas do consumo excessivo e intoxicação. Na verdade, mesmo baixos ou moderados níveis de consumo de álcool podem ter efeitos deletérios, incluindo o desempenho no trabalho, segurança no trânsito e risco de desenvolvimento de DCNTs ${ }^{1}$.

Por fim, os resultados do presente estudo chamam a atenção por que se trata de uma categoria de trabalhadores que apresenta diversos comportamentos de risco para o desenvolvimento de agravos à saúde, tais como jornada de trabalho estressante, hábitos alimentares não saudáveis, precariedade de autocuidados e baixo índice de busca de assistência em saúde ${ }^{13-17}$.

Em relação às DCNTs, o maior risco esteve presente em motoristas que consumiam bebidas alcoólicas em níveis problemáticos (AUDIT $\geq 8$ ), além de apresentarem sintomas sugestivos de doenças como hipertensão arterial severa, Diabetes Mellitus e índices de colesterol acima do esperado. Entre os fatores de risco foi identificada associação entre AUDIT $\geq 8$, sobrepeso e obesidade, conforme a Tabela 3. Sabe-se que um dos maiores desafios para a saúde pública para os próximos anos é controlar o avanço da obesidade em curso no país, que já afeta $16,9 \%$ das mulheres e $12,4 \%$ dos homens adultos brasileiros ${ }^{5,7}$.

Segundo a literatura, os riscos para a saúde associados ao uso agudo e crônico de álcool são diferentes. Por exemplo, um indivíduo que consome 14 doses, em uma única ocasião, apresenta maior probabilidade de envolvimento em acidentes de trânsito, enquanto que um indivíduo que consome mais de duas doses diariamente tem maior risco de desenvolvimento de DCNTs ${ }^{2,6}$.

\section{ConClusão}

Este estudo permitiu identificar o uso do álcool e as condições de saúde em motoristas de caminhão participantes de uma campanha de saúde na estrada. Os resultados mostraram fatores de risco que podem ser modificados por meio de estratégias de prevenção e promoção de saúde voltadas para essa população.
Os problemas associados ao uso do álcool e às condições de saúde, principalmente as DCNTs, atingiram níveis preocupantes nos últimos anos, contribuindo de maneira consistente para o desenvolvimento de diversas doenças, precariedade nas condições de saúde, envolvimento em comportamentos de alto risco, desencadeamento de doenças mentais e acidentes de transportes. Os acidentes de transportes já ocupam o posto de uma das principais causas de mortalidade no mundo.

Dada a magnitude dos riscos associados ao uso de álcool e às doenças crônico-degenerativas, torna-se imperativo repensar estratégias de prevenção e promoção de saúde de forma continuada e sustentável, visando a ampliar o conhecimento sobre os fatores de risco para doenças e agravos não transmissíveis e, como consequência, levar à modificação do estilo de vida.

Uma das limitações do presente estudo referese ao delineamento metodológico utilizado (estudo transversal). Estudos futuros devem contemplar a necessidade de pesquisas com desenhos longitudinais, bem como avaliar a eficácia da utilização de outros recursos para identificação do uso de álcool por motoristas, como o teste do bafômetro. Outra limitação refere-se ao fato de que, na campanha de saúde na estrada, os motoristas de caminhão participaram voluntariamente. Desse modo, torna-se plausível pensar que pode ter havido uma parcela dessa população, que utiliza o sistema rodoviário, que consumia bebidas alcoólicas e não foi avaliada. Também se sugere o desenvolvimento de estudos que avaliem o possível consumo de outras drogas, tais como anfetamina, cocaína e crack.

\section{REFERÊNCIAS}

1.Roerecke M, Haydon E, Giesbrecht N. Alcohol and chronic disease: an Ontario perspective. Alcohol Policy Network. Ontario (Canada): Ontario Public Health Association; 2007.

2.World Health Organization. Global status report on alcohol and health. 2011. Geneva (Swi): WHO; 2011.

3.World Health Organization. Alcohol and injury in emergency departments. Geneva (Swi): WHO; 2007.

4.Malta DC, Silva MMA, Lima CM, Soares Filho AM, Montenegro MMS, Mascarenhas MDMM, et al. Impacto da legislação restritiva do álcool na morbimortalidade por acidentes de transporte terrestre - Brasil, 2008. Epidemiol Serv Saúde. 2010; 19(1):77-8.

5.Rehm J, Giesbrecht N, Patra J, Roerecke M. Estimating chronic disease deaths and hospitalizations due to alcohol use in Canada in 2002: implications for policy and prevention strategies. Prev Chronic Dis [serial online] 2006 Oct [cited 2014 Sep 14]. Available from: http:// www.cdc.gov/pcd/issues/2006/oct/05_0009.htm.

6.World Health Organization. Global status report on road safety 2013: supporting a decade of action. Geneva (Swi): WHO; 2013. 
7.Alwan A. World Health Organization. Global status report on noncommunicable diseases 2010. WHO; 2010.

8.McGovern TF, White WL. Alcohol problems in The United State: twenty years of treatment perspective. New York (USA): The Haworth Press; 2002.

9.Behrendt S, Bühringer G, Perkonigg A, Lieb R, BeesdoBaum K. Characteristics of developmentally early alcohol use disorder symptom reports: a prospective-longitudinal community study. Drug and Alcohol Dependence. 2013; 131:308-15.

10.Jora NP, Magalhães TR, Domingos JBC, Pillon SC. Avaliação do padrão de consumo de álcool e do estresse em motoristas. Rev Eletr Enf. 2010; 12(1). 37-46.

11. Oliveira LG, Yonamine M, Andreucceti G, Ponce JC, Leyton V. Alcohol and other drug use by Brazilian truck drivers: A cause for concern? Rev Bras Psiquiatr. 2012; 34:116-7.

12.Domingos JBC, Pillon SC. O uso do álcool entre motoristas no interior do Estado de São Paulo. Rev enferm UERJ. 2007; 15: 393-9.

13.Pechansky F, Duarte PCAV, De Boni R, organizadores. Uso de bebidas alcoólicas e outras drogas nas rodovias brasileiras e outros estudos. Porto Alegre (RS): Secretaria Nacional de Políticas sobre Drogas; 2010.

14. Yonamine M, Sanches LR, Paranhos BAPB, Almeida RM, Andreuccetti G, Leyton V. Detecting alcohol and illicit drugs in oral fluid samples collected from truck drivers in the State of São Paulo, Brazil. Traffic Injury Prevention. 2013; 14:127-31.

15.Amatuzi Filho C. A saúde do caminhoneiro em debate: fatos e evidências. In: Confederação Nacional de Transporte. Debate em foco, seção saúde do caminhoneiro. 2004. [citado em 14 set 2014]. Disponível em: http//:www.cnt.org.br.

16.Nascimento EC, Nascimento E, Silva JP. Uso de álcool e anfetaminas entre caminhoneiros de estrada. Rev Saúde Pública. 2007; 41:290-3.

17.Domingos JBC, Jora NP, Pimenta AM, Pillon SC. Consumo de álcool, sobrepeso e obesidade entre caminhoneiros. Rev enferm UERJ. 2010; 18:377-82.

18.Babor TF, Higgins-Biddle JC, Saunders JB, Monteiro MG. AUDIT - The Alcohol Use Disorders Identification Test: guidelines for use in primary care. $2^{\mathrm{a}} \mathrm{ed}$. Genebra (Swi): World Health Organization, Department of Mental Health and Substance Dependence; 1992. 